\title{
Anemia postrasplante renal, su efecto en la función y en la sobrevida del injerto
}

\author{
Zavaleta -Martínez Mariana*, Chew-Wong Alfredo**, Rosas-Cabral Alejandro***
}

\begin{abstract}
Resumen
- Introducción: La anemia postrasplante renal (APTR), es una

- entidad con una frecuencia muy variable, y cuya presencia

- puede afectar la función y la sobrevida del injerto. Objetivo:

- Determinar si existe relación entre la presencia de APTR y

- la falla o la pérdida del injerto renal en los pacientes some-

- tidos a trasplante renal en el Centenario Hospital Miguel

- Hidalgo de la Ciudad de Aguascalientes. Materiales y mé-

- todos: Análisis retrospectivo de todos los pacientes mayores

- de 16 años, que recibieron trasplante renal desde enero

- 2010 a diciembre 2014 en nuestro servicio. Se utilizó es-

- tadística descriptiva, análisis de sobrevida de Kaplan Meier

- con prueba de log Rank, y regresión logística múltiple. Una

- $p<0.05$ fue considerada significativa. Resultados: Realiza-

- mos 387 trasplantes renales en el periodo de estudio, e

- incluimos 333 pacientes. La mediana de seguimiento fue

- de 38 meses ( $r=1-6$ I). 43 (I 2.9\%) pacientes desarrollaron

- APTR. Observamos que los pacientes con anemia elevaron

- significativamente sus valores de creatinina después del año

- del trasplante, lo cual traduce la presencia de falla o pérdi-

- da del injerto en la población con anemia. $(p=0.00$ I). La

- mediana de sobrevida del injerto, en pacientes con APTR

- fue de 26 meses, la cual fue significativamente menor a

- la de los pacientes que no presentaron APTR (mediana 49

- meses, $p=0.00$ l). La mediana de sobrevida global de los pa-

- cientes sin APTR, fue significativamente superior (6I meses)

- versus la de los pacientes con APTR (mediana 42 meses,

- $p=0.001$ ). En conclusión nosotros encontramos que existe

- una asociación significativa entre la presencia de APTR y

- la falla o pérdida del injerto. LUXMÉDICA, AÑO 13, NÚMERO 39,

SEPT-DIC 2018, PP 19-28.
\end{abstract}

Palabras clave: anemia postrasplante, pérdida del injerto, falla del injerto.

\section{Abstract}

Introduction: Renal posttransplantation anemia (RPTA) is an entity with a variable frequency, and whose presence can affect the function and survival of the graft. Objective: To determine if there is a relationship between RPTA and the failure or loss of the renal graft in patients undergoing kidney transplantation at the Centenario Hospital Miguel Hidalgo of the city of Aguascalientes. Methods: Retrospective analysis of all the patients over 16 years of age who received kidney transplants from January 2010 to December 2014 in our department. Descriptive statistics, Kaplan-Meier survival analysis with the Log-Rank test, and multiple logistic regression were used. A $p<0.05$ was considered significant. Results: We performed 387 kidney transplants during the study period and included 333 patients. The median followup was 38 months $(r=1-61)$. Forty-three (12.9\%) patients developed RPTA. We observed that patients with anemia significantly increased their creatinine values after one year of the transplant, meaning the failure or loss of the graft in the population with anemia $(p=0.001)$. The median survival of the graft in patients with RPTA was 26 months, which was significantly lower than in patients without RPTA (median 49 months, $p=0.001$ ). The median overall survival of patients without RPTA was significantly higher (6I months) than that of patients with RPTA (median 42 months, $p=0.001$ ). Conclusions: We found that there is a significant association between RPTA and the failure or loss of the graft. LUXMÉDICA, AÑO 13, NÚMERO 39, SEPT-DIC 2018, PP 19-28.

$$
:
$$

$$
\text { - }
$$
-

$$
\text { - }
$$

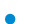
-

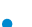
-

$$
\text { - }
$$
$\bullet$ :

(

Keyw Keywords: posttransplantation anemia, graft loss, graft failure

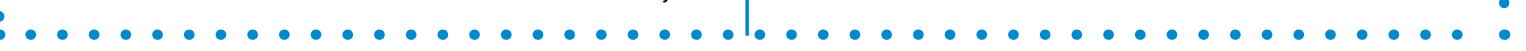

\footnotetext{
* Médico Adscrito al Departamento de Medicina Interna, Centenario Hospital Miguel Hidalgo. Aguascalientes, Aguascalientes. México.

** Jefe del Servicio de Nefrología, Departamento de Medicina Interna, Centenario Hospital Miguel Hidalgo. Aguascalientes, Aguascalientes. México

*** Profesor investigador del Departamento de Medicina. Universidad Autónoma de Aguascalientes. México.
}

Fecha de recibido: 10 de marzo 2018

Fecha de aceptación: 4 de junio 2018

Autor para correspondencia: Dr. Alejandro Rosas Cabral. Departamento de Medicina, Edificio 107, planta alta. Ciudad Universitaria. Universidad Autónoma de Aguascalientes. Avenida Universidad \#940. Código postal 20131. Aguascalientes, Aguascalientes, México. Teléfono 4499108440, correo electrónico: drrosascabral@gmail.com 


\section{Introducción}

La anemia es una complicación frecuente en los pacientes sometidos a trasplante renal, con una frecuencia reportada en la literatura entre el 38 y $40 \%$. Su presencia se ha relacionado con diversos factores como son: la función renal, deficiencia de hierro, inflamación, pérdidas sanguíneas gastrointestinales, síndrome urémico-hemolítico, e infección por parvovirus B19. Así mismo, los pacientes sometidos a trasplante renal reciben tratamiento con diversos fármacos inmunosupresores que, por sus efectos anti-proliferativos, pueden conducir a una menor respuesta eritropoyética de la médula ósea con aparición o bien agravamiento de la anemia., ${ }^{1,2}$ La anemia es un factor de riesgo cardiovascular potencialmente corregible, por la que detectarla a tiempo y saber sobre su impacto en la falla del injerto a corto, mediano y largo plazo es de relevancia. ${ }^{3-5}$

Las distintas definiciones de anemia en este contexto, no han permitido establecer a partir de qué grado de la misma debe iniciarse tratamiento. Algunos autores han establecido una hemoglobina menor a $11.5 \mathrm{~g} / \mathrm{dL}$ para ambos sexos y otros más con hemoglobina menor a $13 \mathrm{~g} / \mathrm{dL}$ en caso del sexo masculino y $12 \mathrm{~g} / \mathrm{dL}$ para pacientes del sexo femenino. Incluso se ha definido anemia postrasplante en aquellos pacientes con hematocrito menor a $36 \%{ }^{6,7} \mathrm{La}$ definición de anemia fue acuñada por la Organización Mundial de la Salud (OMS) en 1964, y se refiere a concentraciones de hemoglobina $(\mathrm{Hb})$ en mujeres $<12 \mathrm{~g} / \mathrm{dL}$ y en los hombres $<13 \mathrm{~g} / \mathrm{dL}$. No obstante, esta definición fue adoptada por la Sociedad Americana de Trasplantes (AST) hasta el año $2001 .^{8}$

Sin tomar en cuenta el tiempo que transcurre entre el trasplante renal y el desarrollo de la anemia, el paciente tiene, durante el mismo evento quirúrgico, pérdidas hemáticas, lo cual favorece la progresión hacia la anemia. Además, independientemente de la recuperación de la función renal, el injerto trasplantado no va a conseguir una producción de eritropoyetina endógena que origine una respuesta eritropoyética eficaz hasta el final del primer mes posterior al trasplante..$^{9,10}$

Un estudio efectuado por Iwamoto y colaboradores concluyó que el mejoramiento de la cifra de hemoglobina en pacientes trasplantados renales se lleva a cabo en promedio a los tres meses, existiendo una correlación significativa entre anemia postrasplante y la función del injerto renal. La función del injerto renal es más baja cuando los niveles de hemoglobina son menores de $11 \mathrm{~g} / \mathrm{dl}$, sin embargo en receptores renales jóvenes una buena función renal, tiene una influencia positiva en el mejoramiento de la anemia. ${ }^{11,12}$

Similar a lo reportado en pacientes en diálisis, las enfermedades cardiovasculares son la principal causa de muerte en pacientes que han recibido un trasplante renal. Por lo que, teniendo en cuenta que la presencia de anemia está asociada con riesgo cardiovascular temprano en pacientes con diabetes, al igual que su presencia es un 
predictor independiente de hipertrofia del ventrículo izquierdo y de insuficiencia cardiaca congestiva; entonces se puede considerar que la presencia de anemia puede ser un predictor negativo en el pronóstico cardiovascular de los pacientes después del trasplante. ${ }^{13}$

Se ha descrito que además de una causa potencial de enfermedad cardiovascular, la anemia también puede contribuir a una mayor y más rápida pérdida de la función renal en pacientes con función renal alterada, prediciendo significativamente la disminución en la tasa de filtración glomerular. ${ }^{13}$

Debido a que existen diferentes estándares establecidos según los centros donde se han realizado los estudios que buscan conocer el impacto de la presencia de anemia y los diferentes desenlaces en el paciente con trasplante renal, hemos desarrollado el presente trabajo para conocer si existe alguna relación entre la presencia de anemia posterior a trasplante renal con la falla del injerto o bien la pérdida del injerto renal en nuestros pacientes.

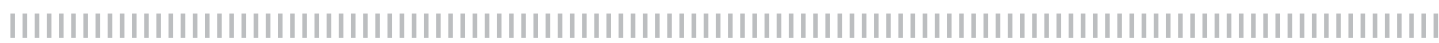

\section{Material y métodos}

Se realizó un estudio retrospectivo, comparativo, y longitudinal, siendo nuestro universo de estudio, pacientes mayores de 16 años, a quienes se les realizó trasplante renal en el Centenario Hospital Miguel Hidalgo, de la Ciudad de Aguascalientes, en el periodo comprendido del $1^{\circ}$ de enero de 2010 al 31 de diciembre de 2014. Se excluyeron: a) pacientes con anemia atribuida a otra enfermedad, b) pacientes con anemia secundaria a medicamentos, c) pacientes con anemia por sangrado de tubo digestivo; d) pacientes cuyos expedientes no proporcionaran la información necesaria para los fines del estudio.

Se realizó una revisión sistemática de los expedientes y se organizaron los datos a través de una hoja de procesamiento de datos con las siguientes variables: edad, sexo, peso, talla, índice de masa corporal, fecha de diagnóstico de insuficiencia renal crónica, tipo de tratamiento renal sustitu- tivo previo, hemoglobina al ingreso hospitalario, a los seis meses, al año y estatus actual; creatinina sérica al ingreso hospitalario, a los seis meses, al año y estatus actual, y el diagnóstico de diabetes mellitus previo al trasplante. Se consideró anemia como el nivel de hemoglobina al año posterior al trasplante menor a $12 \mathrm{~g} / \mathrm{dL}$ para ambos sexos; se definió falla del injerto como una cifra de creatinina sérica mayor o igual a $1.5 \mathrm{mg} / \mathrm{dL}$ posterior al trasplante renal; y pérdida del injerto una creatinina sérica mayor a $2 \mathrm{mg} / \mathrm{dL}$ en el periodo postrasplante, no se consideró para estos criterios la necesidad de reestablecer diálisis o un nuevo trasplante. Se realizaron prueba exacta de Fisher, prueba de $t$ de Student no pareada, análisis de sobrevida de Kaplan-Meier con prueba de log Rank y regresión logística múltiple, mediante el uso del programa Statistics. Un valor de $p$ $<0.05$ fue considerado como significativo.

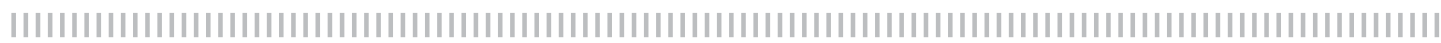

\section{Resultados}

En el periodo de estudio realizamos 387 trasplantes renales en nuestro centro, de los cuales se incluyeron 333 que cumplieron los criterios de inclusión. Predomi- nó el sexo masculino, con 234 pacientes $(70.3 \%)$ y 99 del sexo femenino $(29.7 \%)$, por otro lado 53 pacientes $(16.4 \%)$ tuvieron diagnóstico de diabetes mellitus previo al trasplante renal. La mediana de segui- 
miento de los pacientes fue de 38 meses con rango de uno a 61 meses.

De los diferentes años que abarcó el estudio, el mayor número de trasplantes se realizó en el año 2011, con un total de 95 trasplantes, correspondientes al $29.5 \%$ de los mismos. La edad promedio de los donadores fue de $34 \pm 8.9$ años, en tanto que la edad promedio de los receptores renales fue de $28.1 \pm 13$ años.

La mayoría de los trasplantes se efectuaron en pacientes originarios de nuestra ciudad, representando el $82 \%$ de los trasplantes renales realizados en nuestro centro. Seguidos de pacientes originarios de Guanajuato (6\%), y en tercer lugar pacientes originarios de Zacatecas (6\%), el resto fueron originarios de diferentes poblaciones de nuestro estado. El tipo de trasplante más frecuente fue el de donador vivo relacionado, representando el $83 \%$ del total de los trasplantes realizados en el periodo de estudio, seguido por el de donador cadavérico, con un $9 \%$. El $37 \%$ de los pacientes receptores renales tenían sobrepeso previo al trasplante renal (119 pacientes). De los 333 pacientes incluidos en el estudio en 43 $(12.9 \%)$ se desarrolló anemia postrasplante renal y en 290 (87.1\%) no (tabla 1$)$.

Las causas identificadas como factores que influyeron al desarrollo de la insuficiencia renal, en su mayoría fueron desconocidas, solamente lográndose diagnosticar mediante estudio histopatológico la glomerulopatía primaria como causa de la enfermedad renal crónica en 37 de los pacientes $(11.4 \%)$.

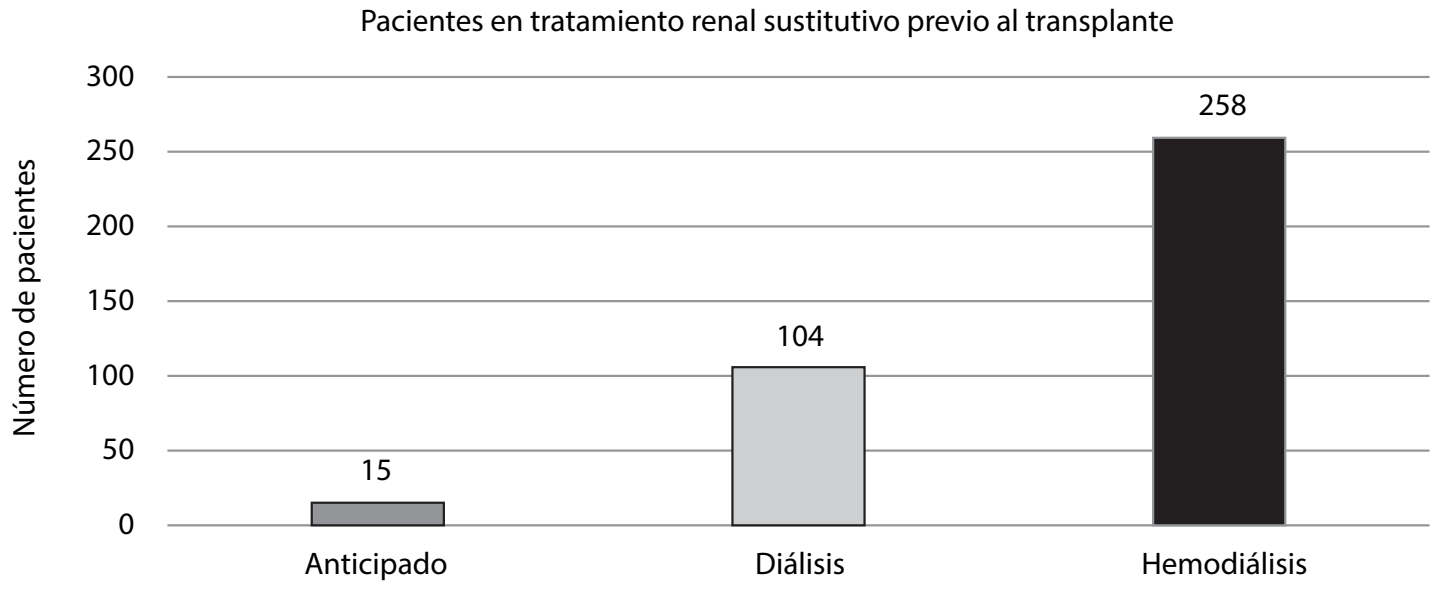

Gráfica 1. Pacientes en tratamiento renal sustitutivo previo al trasplante en 333 pacientes llevados a trasplante renal en el Centenario Hospital Miguel Hidalgo de la Ciudad de Aguascalientes.

En cuanto al modo de tratamiento de sustitución renal previo a trasplante, el modo más empleado fue hemodiálisis, con 258 pacientes (80.1\%). Realizándose trasplante renal anticipado en 15 pacientes (4.6\%) (gráfica 1). 


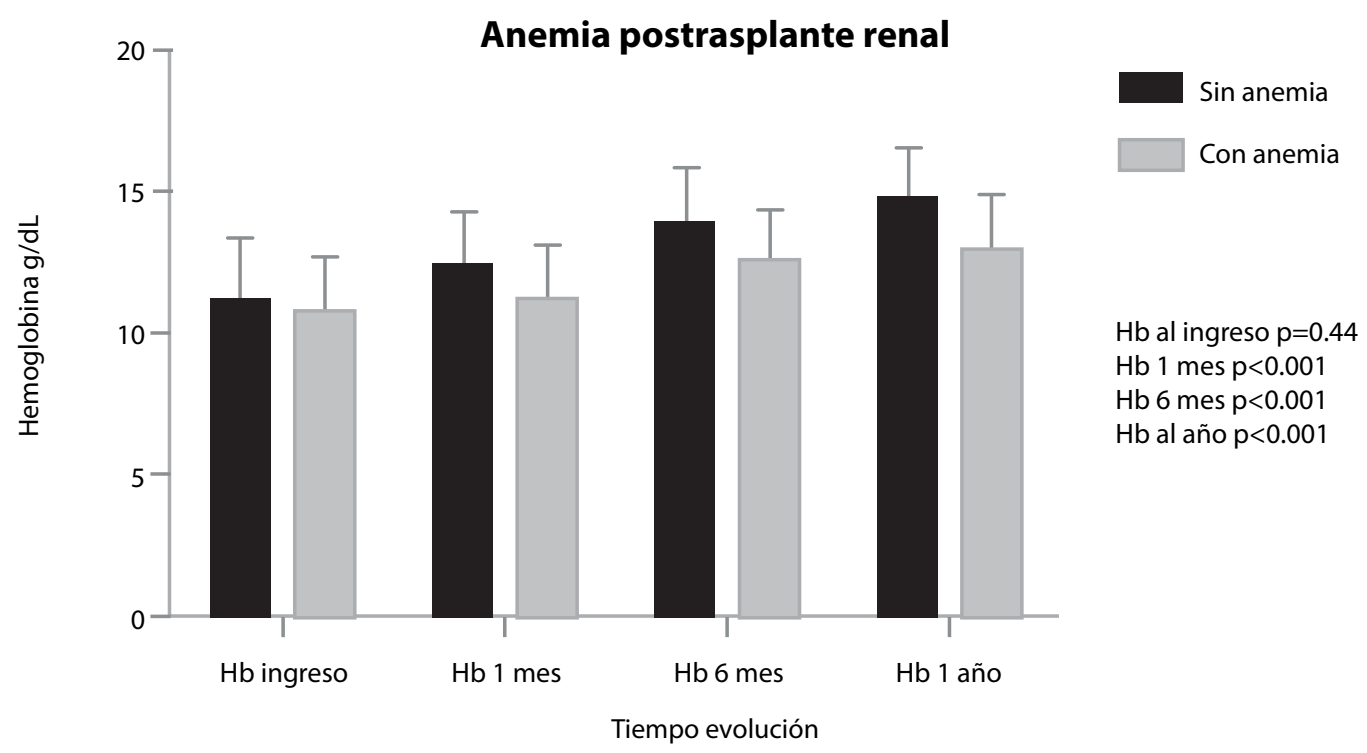

Gráfica 2. Comportamiento de la cifra de hemoglobina en un grupo de 333 pacientes llevados a trasplante renal en el Hospital Miguel Hidalgo de la Ciudad de Aguascalientes.

No encontramos diferencia significativa entre la cifra de hemoglobina al momento del ingreso del paciente para realizar trasplante renal entre ambos grupos de pacientes, pero sí se observa un incremento significativo en la cifra de hemoglobina al mes, a los seis meses y al año posterior a la intervención quirúrgica, lo cual traduce la mejoría en la función renal y su impacto en la producción de eritropoyetina desde el mes del trasplante (gráfica 2).

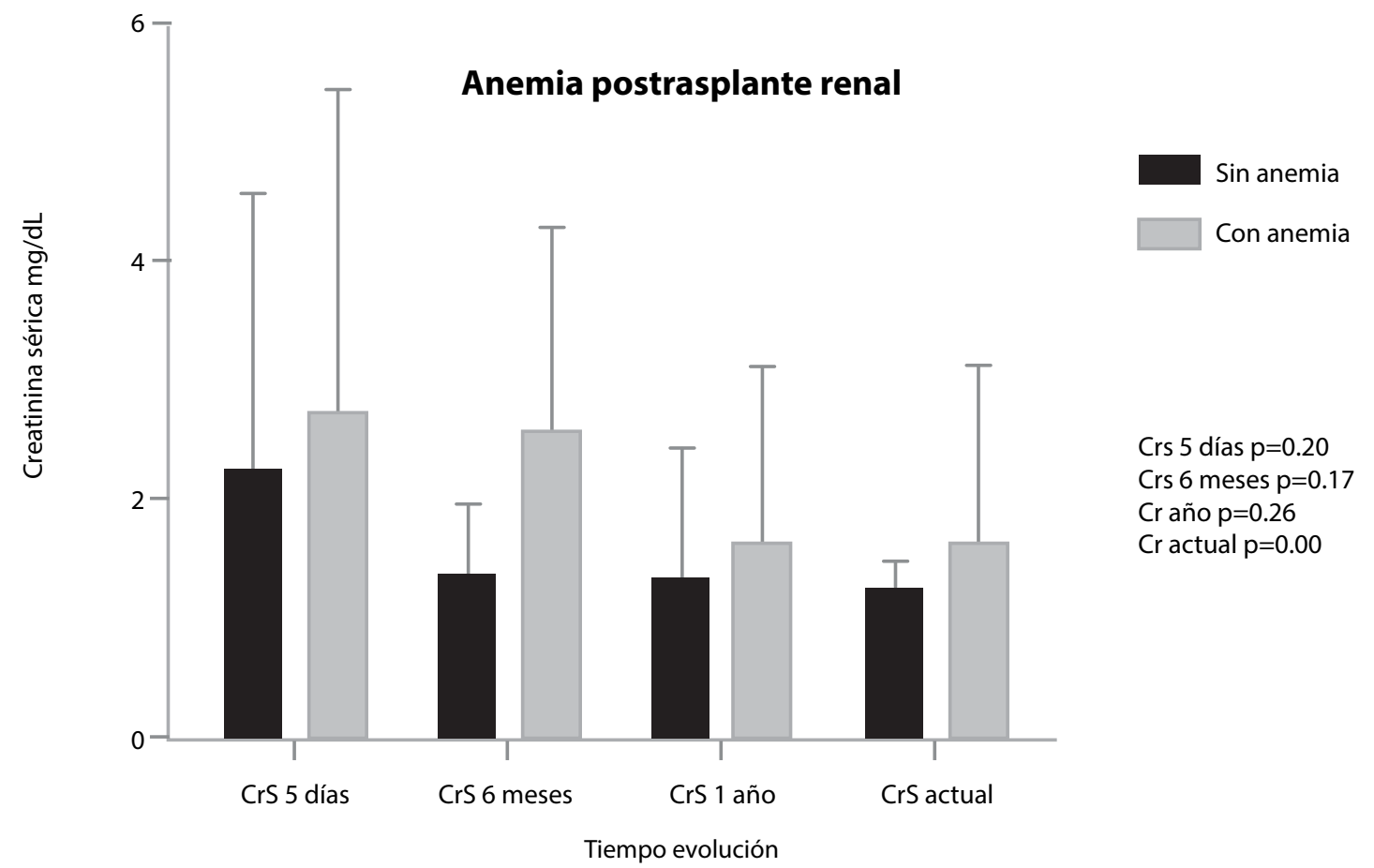

Gráfica 3. Función renal y presencia de anemia postrasplante renal en 333 pacientes llevados a trasplante renal en el Centenario Hospital Miguel Hidalgo de la Ciudad de Aguascalientes. 
Se puede observar que el nivel de creatinina sérica no es estadísticamente diferente entre los grupos de pacientes con anemia o sin anemia, a los 5 días, seis meses y un año posterior al trasplante; no obstante, posterior a este momento, los pacientes con anemia elevan significativamente sus valores de creatinina, lo cual traduce la presencia de falla o pérdida del injerto en la población con anemia. De la misma manera el ser mujer se asoció significativamente con mayor riesgo de anemia postrasplante al año $(p=0.001)$ (gráfica 3).

\section{Tabla I}

\section{Presencia de anemia posterior a trasplante renal en $\mathbf{3 3 3}$ pacientes llevados a trasplante en el Centenario Hospital Miguel Hidalgo.}

\begin{tabular}{|c|c|c|c|}
\hline Características de los pacientes & $\begin{array}{l}\text { Sin APTR } \\
(n=290)\end{array}$ & $\begin{array}{c}\text { Con APTR } \\
(n=43)\end{array}$ & $\mathbf{P}$ \\
\hline Edad ( años) & $27 \pm 12$ & $27 \pm 15$ & 0.99 \\
\hline $\mathrm{IMC}(\mathrm{kg} / \mathrm{m} 2)$ & $23.1 \pm 4.5$ & $22 \pm 4.3$ & 0.73 \\
\hline $\mathrm{CrS}$ al egreso del trasplante $(\mathrm{mg} / \mathrm{dl})$ & $2.1 \pm 2.35$ & $2,7 \pm 2.7$ & 0.20 \\
\hline CrS a los 6meses (mg/ dl) & $1.3 \pm 0.65$ & $1.9 \pm 2.3$ & 0.17 \\
\hline CrS 1 año ( mg/dl ) & $1.3 \pm 1.1$ & $1.6 \pm 1.5$ & 0.26 \\
\hline $\mathrm{CrS}$ al final del seguimiento $(\mathrm{mg} / \mathrm{dl})$ & $1.23 \pm 0.25$ & $1.6 \pm 1.5$ & 0.001 \\
\hline Género femenino (\%) & 27.5 & 53 & 0.001 \\
\hline Hemoglobina al mes $(\mathrm{g} / \mathrm{dL})$ & $12,6 \pm 1.7$ & $10.8 \pm 1.6$ & 0.001 \\
\hline Hemoglobina a 6 meses $(\mathrm{g} / \mathrm{dL}$ ) & $13.96 \pm 1.97$ & $10.57 \pm 1.83$ & 0.001 \\
\hline
\end{tabular}

$A P R T=$ Anemia postrasplante renal, $I M C=$ Índice de masa corporal, $C r S=$ Creatinina sérica.

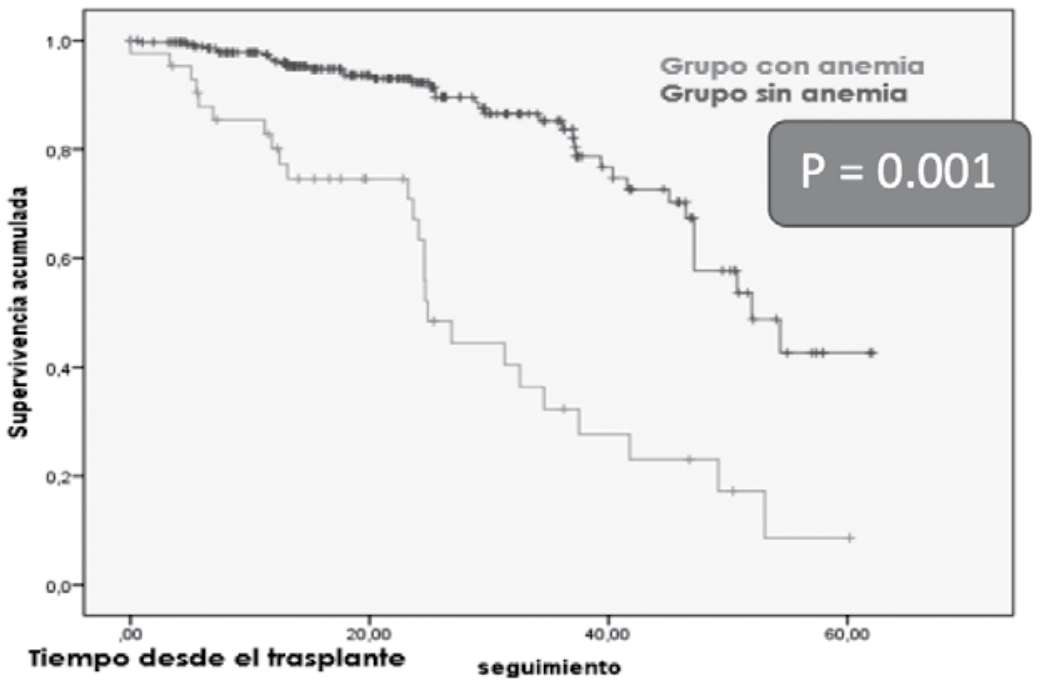

Figura 1.- Comparación en la supervivencia del injerto en un grupo de 333 pacientes llevados a trasplante renal en el Centenario Hospital Miguel Hidalgo de la ciudad de Aguascalientes con o sin anemia postrasplante renal. 
La mediana de sobrevida del injerto, en aquellos pacientes que presentan anemia posterior al trasplante renal fue de 26 meses, la cual es significativamente menor a la de los pacientes que no presentaron anemia postrasplante renal, quienes tuvieron una mediana de sobrevida de 49 meses $(p<0.001)$ (figura 1).

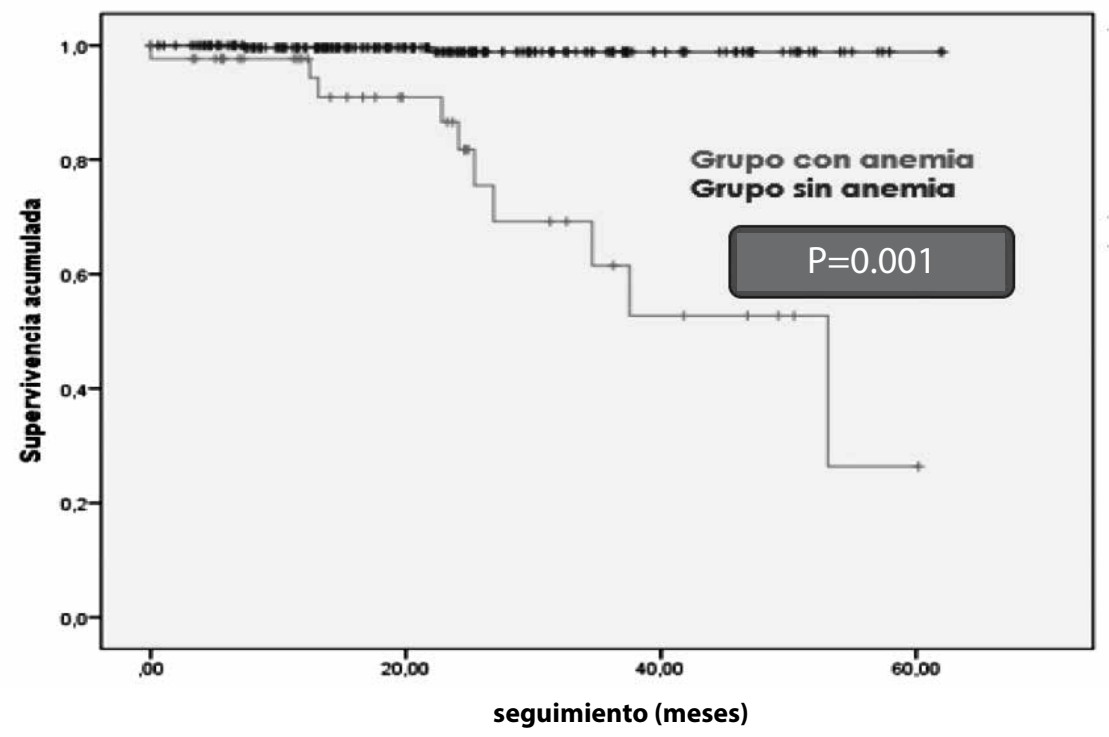

Figura 2.- Anemia al final del seguimiento y sobrevida global del paciente trasplantado en 333 pacientes con trasplante renal en el Centenario Hospital Miguel Hidalgo de la Ciudad de Aguascalientes.

La mediana de la sobrevida global de los pacientes trasplantados sin anemia, fue significativamente superior (61 meses) versus la de aquellos pacientes trasplantados con anemia, los cuales presentaron una mediana de sobrevida de 42 meses $(p<0.001)$ (figura 2$)$. Cabe señalar que el promedio de función renal mediante la fórmula CKD-EPI en pacientes sin anemia postrasplante fue de $92 \mathrm{ml} / \mathrm{min} / \mathrm{m}^{2}$, mientras que el promedio de la función renal en pacientes con anemia postrasplante fue de $45 \mathrm{ml} / \mathrm{min} / \mathrm{m}^{2}$.

Se encontró una relación estadísticamente significativa entre la presencia de anemia al año posterior al trasplante y la falla renal al final del seguimiento $(p=0.001)$. De la misma manera la pre- sencia de anemia al sexto mes posterior al trasplante, se asoció con la pérdida del injerto al año $(p=0.001)$ y a falla del injerto al año $(p=0.001)$. Encontramos una relación significativa entre la falla del injerto y la no adherencia al seguimiento de las consultas $(p=0.001)$.

No encontramos relación entre el diagnóstico de diabetes mellitus con la presencia de anemia al final del seguimiento $(p=0.78)$. Como tampoco se encontró relación entre el sobrepeso previo al trasplante renal con el desarrollo de anemia al final del seguimiento $(p=0.49)$.

Durante el periodo del estudio se llevaron a cabo 27 biopsias del injerto renal, de las cuales en el $72 \%$ de ellas reporte histopatológico fue rechazo humoral agudo. 


\title{
Tabla 2
}

\begin{abstract}
Análisis multivariado en $\mathbf{3 3 3}$ pacientes sometidos a trasplante renal en el Centenario Hospital Miguel Hidalgo de la Ciudad de Aguascalientes.
\end{abstract}

\begin{tabular}{|lcc|}
\hline Variable de desenlace & Razón de momios & P \\
\hline Falla del injerto & 6.00 & 0.001 \\
\hline Pérdida del injerto & 2.98 & 0.033 \\
\hline
\end{tabular}

En el análisis multivariado para conocer si la presencia de anemia afecta a alguno de los desenlaces terminales de la función renal en pacientes que reciben trasplante renal, encontramos que la presencia de la misma otorga un RR de seis veces la posibilidad de falla del injerto siendo esto altamente significativo $(p<0.001)$ y un RR de 2.98 para la pérdida del injerto pero con menor significancia estadística (tabla 2). En el análisis multivariado para determinar los factores que influyen con la presencia de anemia postrasplante al año, un IMC > 25 $y$ un incremento en la cifra de hemoglobina al primer mes posterior al evento quirúrgico, fueron factores protectores para el desarrollo de anemia postrasplante $(p=$ $<0.01)$ y $(p=0.001)$ respectivamente; en contraparte la pérdida del injerto por otras causas fue un factor de riesgo independiente que se asoció al desarrollo de anemia postrasplante $(p=0.001)$.

\section{| | | | | | | | | | | | | | | | | | | | | | | | | | | | | | | | | | | | | | | | | | | | | | | | | | | | | | | | | | | | | | | | | | | | | | | | | | | | | | | | | | | | | | | | | | | | | | | | | | | | | | | | | | | | | | | |}

\section{Discusión}

En este estudio exploramos la posibilidad de la existencia de una relación entre el desarrollo de anemia en el periodo posterior al trasplante renal y la pérdida o falla del injerto después de un periodo largo de seguimiento, conociendo que existen pocos estudios al respecto. Partiendo incluso con el hecho de que existen múltiples criterios en la literatura para definir la presencia de anemia postrasplante renal y que a pesar de que desde 2009, las guías recomiendan el tratamiento de la anemia en el paciente con trasplante renal, según la causa subyacente, en las mismas no se recomienda un monitoreo de la anemia, e incluso las guías están basadas en los criterios de tratamiento para los pacientes en el periodo dialítico y no existen guías específicas del tratamiento en el periodo posterior al trasplante. Nuestros resultados sugieren que el nivel sérico de hemoglobina o bien, la presencia de anemia, están independiente y significativamente asociados con la falla del injerto, al igual que a la pérdida del injerto.
De la misma manera encontramos que la presencia de anemia está asociada con la supervivencia y la falla del injerto en nuestros pacientes receptores de trasplante renal. Es decir los pacientes con anemia tienen menor probabilidad de sobrevivir que los pacientes sin anemia, similar a lo reportado por Majernikova y colaboradores. ${ }^{20}$

En el análisis multivariado encontramos una asociación significativa entre el nivel sérico de hemoglobina mayor de $12 \mathrm{~g} / \mathrm{dL}$ y la sobrevida. A diferencia de lo reportado por Winkelmayer y colaboradores, quienes tomaron como referencia para definir anemia un nivel de hemoglobina inferior a 10 $\mathrm{g} / \mathrm{dL}$, lo cual les resultó en pocos eventos para analizar y por consiguiente un bajo poder estadístico; ${ }^{14}$ pero concordante a lo descrito por Molnar y colaboradores, así como Bayound y cols, quienes encontraron una asociación significativa entre el nivel de hemoglobina y la supervivencia a 46 meses de seguimiento. 1,13,15 Así mismo, nuestros resultados no difieren de lo reportado a seis o 12 meses por Locatelli 
y colaboradores en donde la presencia de anemia se relaciona significativamente con falla renal o pérdida del injerto, ${ }^{16}$ así como en el trabajo de Huang y colaboradores, donde la presencia de anemia a los $12 \mathrm{me}$ ses posterior al trasplante se relacionó significativamente con falla del injerto. ${ }^{17}$

Por otro lado, Bayound y colaboradores reportaron una relación significativa entre la presencia de sobrepeso y la de anemia posterior al trasplante, sin embargo en el análisis multivariado de nuestros pacientes nosotros encontramos que la presencia de sobrepeso es un factor protector para el desarrollo de anemia posterior al trasplante. Se requieren más estudios al respecto para determinar, si el sobrepeso es o no un factor predictor de anemia en estos pacientes. ${ }^{15}$
Con lo anterior podemos sugerir que como parte del manejo del paciente trasplantado renal que desarrolla anemia, se agregue al tratamiento eritropoyetina y hierro, para con esto elevar el nivel de hemoglobina y así intentar prolongar la sobrevida y supervivencia tanto del injerto como del paciente trasplantado. Acorde a lo referido por Spiegel y Chertow, sin embargo recientemente se ha referido en la literatura que esta estrategia de tratamiento se asocia a un incremento en la tasa de trombosis y progresión a cáncer, aunado a que todavía falta definir con mayor certeza el nivel de hemoglobina al que hay que llevar a los pacientes con esta estrategia de tratamiento sin elevar el riesgo de complicaciones como las previamente mencionadas. $^{18,19}$

\section{Conclusiones}

Encontramos que en nuestros pacientes, sí existe una asociación significativa entre la presencia de anemia postrasplante y la falla o pérdida del injerto; de la misma manera la sobrevida del injerto, así como la supervivencia global del paciente trasplantado, es significativamente mayor en aquellos pacientes que no desarrollan anemia postrasplante renal.

\section{Bibliografía}

1. Alberú J. Mancilla E. Inmunosupresión para receptores de trasplante renal: estrategias actuales. Rev Inv Clin. 2005; 57(2): 213-224.

2. Hricik D.E. Anemia after kidney transplantation -Is the Incidence Increasing? Am J Transplant. 2003; 3(7): 771-772.

3. Moresco F. Alegre R. Ariceta G. Beneyto I. Bernis C. Calvo N, et al. Tratamiento de la anemia postrasplante renal. Nefrología. 2011; 2(2):16-24.

4. Joist H. Brennan D.C. Coyne D.W. Anemia in the kidney transplant patient. Adv Chronic Kidney Dis. 2006; 13 (1): 4-10.

5. Chadban S.J. Baines L. Polkinghome K. Jefferys A. Dogra S. Kanganas C. Irish A. Walker S. Anemia after kidney transplantation is not completely explained by reduced kidney function. Am J Kidney Dis. 2007; 49(2): 301-309.
6. Vanrenterghem Y. Ponticelli C. Morales J.M. Abramowicz D. Baboolal K. et al. Prevalence and management of anemia in renal transplant recipients: $A$ European survey. Am J Transplant. 2003; 3(7): 835845.

7. Yang $Y, Y u$ Y, Chen Y. Blood disorders typically associated with renal transplantation. Front Cell Dev Biol. 2015; 3, 18, doi:10.3389.

8. Baizabal-Olarte R. Morales-Buenrostro L.E. Anemia en receptores de transplante renal. Rev Mex Transpl. 2012; 1(1): 29-37.

9. Yorgin P.D. Scandling J.D. Belson A. Sanchez J. Alexander S.R. Andreoni K.A. Late post-trasplant anemia in adult renal transplant recipients. An underrecognized problem? Am J Transplant. 2002; 2 (5): 429-435.

10. Weir M.R. Is Anemia a predictor for mortality and loss graft function in kidney transplant recipients? Am J Transplat. 2002; 7(4): 731-732. 
11. Iwamoto H. Nakamura Y. Konno O. Hama K. Yokohama T. Kihara Y. Kawachi S. Shimazu M. Correlation between post kidney transplant anemia and kidney graft function. Transplantation Proc. 2014; 46 (2): 496-498.

12. Lorenz M. Kletzmayr J. Perschl A. Furrer A. Hör W.H. Sunder-Plassman G. Anemia and iron deficiencies among long-term renal transplant recipients. J Am Soc Nephrol. 2002; 13(3): 794- 779.

13. Molnar M. Z. Czira M. Ambrus C. Szeifert L. Szentkiralyi $A$. Beko $G$. et al. Anemia is associated with mortality in kidney-trasplanted patients. A prospective cohort study. Am J Transplant. 2007; 7(4): 818-824.

14. Winkelmayer W.C. Kewalramani R. Rutstein M. Gabardi S. Vonvisger T. Chandraker A. Pharmacoepidemiology of anemia in kidney transplant recipients. J Am Soc Nephrol. 2004; 15(5): 1347-1352.

15. Bayoud Y. Kamdoum N. Kamdoum N.M.L. Marchand C. Kozal S. Cholley I. et al. (2014). The impact of obesity on renal function at 1 year after kid- ney transplantation: Single -center experience. Prog Urol. 2014; 24(16): 1063-1068.

16. Locatelli F. Del Vecchio L. Pozzoni P. Anemia and cardiovascular risk: The lesson of the CREATE trial. J Am Soc Nephrol. 2003; 17 (Suppl 3): S262-S266.

17. Huang Z. Song T. Fu L. Rao Z. Zeng D. Qiu Y. et al. (2015). Post-renal transplantation anemia at 12 months: prevalence, risk factors, and impact on clinical outcomes. Int Urol Nephrol. 2015; 47(9):15771585.

18. Spiegel DM, Chertow GM. Lost without directions: lessons from the anemia debate and the drive study. Clin J Am Soc Nephrol. 2009;4(5);1009-1010.

19. Kestenbaum B. Reevaluating erythropoiesis-stimulating agents. N Engl J Med. 2010; 362 (18):1742.

20. Majernikova $M$, Rosenberger J, Prihdova $L$, Jarcuskova M, Roland R, Groothoff JW, van Dijk JP. Postransplat anemia as a prognostic factor of mortality in kidney-transplant recipients. Biomed Res Int. 2017; 2017; 6982240. 